Classification

Physics Abstracts

$0.665-2.220$

\title{
COMPORTEMENT CONTRÔLÉ DE DEUX ENROULEMENTS SUPRACONDUCTEURS GIGOGNES LORS DU RÉTABLISSEMENT DE L'ÉTAT NORMAL
}

\author{
J. L. BRET, G. DeVeley $\left({ }^{*}\right)$, J. P. FAURE et J. C. VAllier $(* *)$
}

Centre de Recherches sur les Très Basses Températures, C. N. R. S., Grenoble, France

(Reçu le 21 mars 1974)

\begin{abstract}
Résumé. - Dans certains bobinages supraconducteurs produisant des champs magnétiques élevés, les énergies magnétiques mises en jeu sont assez importantes pour détériorer quelquefois ces bobinages lors d'une transition.

Pour un ensemble de deux aimants supraconducteurs couplés électromagnétiquement, les auteurs décrivent un procédé simple permettant d'éviter toute détérioration par échauffement lors d'un rétablissement de l'état normal.
\end{abstract}

\begin{abstract}
In some superconducting coils producing high magnetic fields, the magnetic energies brought into play are sometimes important enough to damage these coils during a transition.

For a set of two magnetically connected superconducting electromagnets, the authors describe a simple process which allows one to avoid any damage by heating during a quench.
\end{abstract}

Les aimants supraconducteurs permettent actuellement la production de champs magnétiques supérieurs à $15 \mathrm{~T}$. Il n'est pas nécessaire de disposer d'une puissance électrique de plus de quelques kVA pour les faire fonctionner, ce qui entraîne une utilisation de plus en plus systématique de ce type d'aimants.

Avec l'alliage Nb-Ti employé dès 1965 , on a rapidement pu produire plus de $8 \mathrm{~T}$. La limite actuelle permise avec ce matériau se situe au-delà de $10 \mathrm{~T}$, valeur de champ magnétique accessible avec des bobinages travaillant à $1,8 \mathrm{~K}$ et utilisant des alliages $\mathrm{Nb}$ - $\mathrm{Ti}$ à structure multifilamentaire.

Pour produire des champs magnétiques supérieurs à la dizaine de Tesla, on est amené à employer des matériaux supraconducteurs comme $\mathrm{Nb} 3-\mathrm{Sn}$ ou V3Ga. Deux solutions sont alors choisies indifféremment :

a) on réalise le bobinage avec un seul matériau,

b) on réalise un bobinage mixte dans lequel l'alliage $\mathrm{Nb}$-Ti sera utilisé dans toutes les régions où l'emploi de matériaux à fort champ critique n'est pas indispensable.

La deuxième possibilité, qui n'est évidemment pas la plus simple, est cependant quelquefois retenue en

(*) Actuellement à l'Institut Universitaire Technologique de Saint-Nazaire.

$\left({ }^{*}\right)$ Actuellement Service National des Champs Intenses, Grenoble. raison de son plus faible prix de revient. Dans la limite de cet article, nous nous intéresserons uniquement aux bobinages mixtes, constitués de deux enroulements concentriques emboîtés l'un dans l'autre, et généralement réalisés avec des matériaux supraconducteurs de caractéristique et de dimension différente.

Les enroulements fonctionnent en série ou indépendamment l'un de l'autre, de manière à produire l'induction magnétique la plus élevée.

On retient assez souvent le fonctionnement séparé car il permet d'accroître dans certaines conditions la stabilité de l'aimant ainsi que son niveau de performance.

Dans les aimants mixtes de petite taille (énergies magnétiques mises en jeu de l'ordre de la centaine de kilojoules) les aimants sont le plus souvent «autoprotégés " lors du rétablissement de l'état normal : les sources de courant qui les alimentent sont des générateurs débitant de forts courants sous quelques volts, et la tension aux bornes des aimants étant limitée, les courants décroissent lors des transitions : la vitesse de décroissance des courants est en général telle que le bobinage n'est pas détérioré. Au contraire, dans les plus gros bobinages, un tel type de fonctionnement lors de la transition peut amener de graves dégâts à l'aimant. Il est possible d'éviter ce genre d'accident en transférant une partie plus ou moins importante de l'énergie magnétique stockée dans un élément approprié, extérieur aux bobinages. Ce procédé qui est d'ailleurs employé sur tous les grands aimants de 
chambre à bulle, a été repris au niveau de la réalisation d'un aimant mixte mis au point au CRTBT avec le soutien d'un contrat DGRST [1]. L'aimant fonctionne de manière fiable à $14 \mathrm{~T}$ et n'est pas détérioré par les transitions successives auxquelles il a été soumis. Nous nous attachons à décrire dans cet article, les techniques de protection que nous avons utilisées.

1. Echauffement dans un bobinage supraconducteur lors du rétablissement de l'état normal. - 1.1 CAS D'UN BOBINAGE SIMPLE. - On se place ici dans le cas le plus défavorable, ou à partir du rétablissement initial de l'état normal, dans un élément de longueur de matériau, la propagation du front de chaleur est assez rapide pour que le processus de la transition soit totalement adiabatique. Dans ces conditions, la température atteinte au point le plus chaud, $T_{\mathrm{f}}$, à partir d'une température initiale $T_{\mathrm{i}}$ s'obtient à partir de l'égalité :

$$
\int_{0}^{\infty} J^{2} \mathrm{~d} t=A \int_{T_{i}}^{T_{\mathrm{f}}} \frac{C}{\rho} \mathrm{d} T .
$$

$J$ représente ici la densité de courant dans le cuivre stabilisant le supraconducteur, $\boldsymbol{C}$ la capacité calorifique de ce cuivre, $\rho$ sa résistivité. $A$ est un coefficient de correction tenant compte à la fois du rapport cuivre/supraconducteur du matériau et de la différence de capacité calorifique entre cuivre et supraconducteur. La résistivité du cuivre est supposée très petite, devant celle du supraconducteur à l'état normal, dans toute la gamme de température.

Au moment de la transition, une résistance $R_{\mathrm{b}}$ apparaît dans le bobinage. Cette résistance varie au cours du temps, et si le circuit extérieur à l'aimant a une résistance $R_{\mathrm{e}}$, la décroissance du courant dans le bobinage se fait à une vitesse qui dépend de $R_{\mathrm{b}}+R_{\mathrm{e}}$. Si $R_{\mathrm{b}}$ est petit devant $R_{\mathrm{e}}$, la plus grande partie de l'énergie magnétique stockée est cédée sous forme de chaleur au circuit extérieur. Dans le cas contraire, une fraction importante de l'énergie magnétique stockée dans le bobinage est cédée à celui-ci, son échauffement étant alors fonction de l'énergie magnétique qui s'y trouve convertie en chaleur.

On peut rapidement examiner ces deux cas :

a) $R_{\mathrm{b}} \ll R_{\mathrm{e}}$ : il s'écoule un intervalle de temps $t_{0}$ entre l'apparition de la première zone normale et la décroissance du courant dans le circuit. En appelant $\tau$ la constante de temps $L / \boldsymbol{R}_{\mathbf{c}}$ de ce circuit, $L$ étant la self du bobinage, on peut écrire :

$$
\left\lfloor\frac{\tau}{2}+t_{0}\right] J_{0}^{2}=A \int_{T_{\mathrm{i}}}^{T_{\mathrm{f}}} \frac{C}{\rho} \mathrm{d} T
$$

$J_{0}$, la densité de courant dans le cuivre pour $t=0$ est supposée rester constante jusqu'à $t=t_{0}$.

Cette égalité permet de définir la température maximale atteinte au point le plus chaud du bobinage, en l'absence d'échange de chaleur.

b) $R_{\mathrm{b}} \sim \boldsymbol{R}_{\mathrm{e}}$ : la température réellement atteinte au point chaud ne peut plus se calculer comme précédem- ment, car elle dépendra de la manière dont varie $R_{\mathrm{b}}$ au cours de la transition. $\left(R_{\mathrm{b}}(t)\right.$ sera définie avec précision en décrivant la propagation du front de chaleur à l'intérieur du bobinage.) Mais, le niveau de température atteint au point chaud sera forcément inférieur à celui que permet de calculer l'équation (2) précédente, puisque la décroissance du courant dans le circuit va se faire avec une constante de temps "associée " inférieure en durée à $L / R_{\mathrm{e}}$.

Le niveau d'échauffement dans un aimant supraconducteur dépend donc notamment de la caractéristique de l'alimentation de cet aimant. Plusieurs auteurs ont proposé de réduire l'échauffement maximum dans les aimants supraconducteurs en déconnectant ceux-ci de leurs sources de courant dès l'apparition d'une région normale, et en les commutant sur une résistance de décharge extérieure [2-3-4]. Sans revenir dans le détail sur ce procédé, remarquons que l'on ne peut pas donner à $R_{\mathrm{e}}$ une valeur quelconque, car la surtension qui apparaît aux bornes de l'aimant lorsqu'on l'isole de son alimentation est directement proportionnelle à $\boldsymbol{R}_{\mathrm{e}}$.

1.2 CAS DE DEUX BOBINAGES SUPRACONDUCTEURS COUPLÉS. - Considérons le cas d'un aimant mixte, pour lequel, au moment où commence à apparaître une région normale, des interrupteurs isolent chacun des aimants de sa source de courant en les commutant sur des résistances extérieures de décharge $R_{\mathrm{e} 1}$ et $R_{\mathrm{e} 2}$.

Supposons tout d'abord que les résistances qui apparaissent dans l'un et l'autre aimant sont tout à fait négligeables devant les résistances extérieures associées. Si $i_{1}$ et $i_{2}$ sont les courants circulant dans les circuits (1) et (2) de l'un et l'autre aimant, à un instant quelconque, les énergies Joule dissipées dans les circuits (1) et (2) sont respectivement :

$$
J_{1}=\int_{0}^{\infty} R_{\mathrm{e} 1} i_{1}^{2} \mathrm{~d} t \quad J_{2}=\int_{0}^{\infty} R_{\mathrm{e} 2} i_{2}^{2} \mathrm{~d} t .
$$

Le calcul des courants $i_{1}$ et $i_{2}$ est classique :

$$
\begin{aligned}
& i_{1}=A \exp \left(p_{1} t\right)+B \exp \left(p_{2} t\right) \\
& i_{2}=C \exp \left(p_{1} t\right)+D \exp \left(p_{2} t\right) .
\end{aligned}
$$

Les valeurs de $A, B, C, D, p_{1}$ et $p_{2}$ ne sont pas explicitées ici, mais les courants décroissent plus ou moins rapidement en fonction de divers paramètres, notamment en fonction du rapport $I_{02} / I_{01}$ des courants dans chacun des circuits au moment de l'ouverture des liaisons avec les alimentations, et aussi en fonction du rapport des constantes de temps propres à chacun des circuits, $L_{2} R_{\mathrm{e} 1} / L_{1} R_{\mathrm{e} 2}$. On peut concevoir que pour des raisons technologiques, l'un des enroulements soit plus fragile que l'autre. Il conviendra donc que la quantité d'énergie dissipée dans le circuit de l'aimant le plus fragile soit aussi limitée que possible, à charge pour le circuit de l'autre aimant d'assurer la dissipation d'une quantité d'énergie aussi grande que possible.

L'échauffement maximum au point chaud est pro- 
portionnel à $J_{1} / R_{\mathrm{e} 1}$ dans le premier enroulement, à $J_{2} / R_{\mathrm{e} 2}$ dans le second. En revenant aux définitions précédentes, en appelant $t_{01}$ et $t_{02}$ les intervalles de temps séparant le rétablissement initial de l'état normal de l'ouverture des circuits d'alimentation, $\tau_{1}$ et $\tau_{2}$ les constantes de temps propres à chaque circuit on obtient :

$T_{\mathrm{f} 1}$, température finale au point chaud dans le premier circuit proportionnelle à

$$
\left[t_{01}+\frac{\tau_{1}}{2}\right] \cdot \frac{J_{01}^{2}}{R_{\mathrm{e} 1}}
$$

$T_{\mathrm{f} 2}$, température finale au point chaud dans le deuxième circuit, proportionnelle à

$$
\left[t_{02}+\frac{\tau_{2}}{2}\right] \cdot \frac{J_{02}^{2}}{R_{\mathrm{e} 2}}
$$

On peut assez simplement calculer les valeurs que doivent prendre $R_{\mathrm{e} 1}$ et $R_{\mathrm{e} 2}$ compte tenu du rapport des courants $I_{02} / I_{01}$ pour que $T_{\mathrm{f} 1}$ et $T_{\mathrm{f} 2}$ restent dans des limites raisonnables.

Lorsque les résistances qui apparaissent dans les enroulements au moment de la transition ne sont plus négligeables, devant les résistances correspondantes extérieures, comme nous l'avons fait remarquer précédemment, les températures $T_{\mathrm{f} 1}$ et $T_{\mathrm{f} 2}$ déterminées à l'aide du calcul ci-dessus représentent des valeurs par excès de la température atteinte au point chaud de chaque bobinage. De plus, on peut remarquer que dans un système mixte, un seul des aimants devient normal pour $t=0$, le second aimant devenant souvent normal à son tour plus tardivement. On peut donc dire que le calcul élémentaire décrit ici reste valable pour tous les types d'aimants mixtes, pourvu que $T_{\mathrm{f} 1}$ et $T_{\mathrm{f} 2}$ soient des températures inférieures aux limites de température admissibles dans les matériaux du bobinage.

2. Les techniques de protection. - Dans ce qui précède, on peut remarquer que la température maximale atteinte en cas de transition dans l'un ou l'autre aimant est directement dépendante du délai $t_{01}$ ou $t_{02}$ que l'on s'accorde pour isoler les bobinages de leur alimentation respective. Il est en effet connu que les enroulements supraconducteurs sont souvent le siège de tensions électriques développées de manière intermittente, caractérisant ou le phénomène du saut de flux, ou quelquefois, plus banalement, le déplacement d'une spire soumise à un effort électromagnétique intense.

Il convient de ne pas commander intempestivement le déclenchement des dispositifs de protection alors que la tension électrique décelée ne traduit pas forcément le rétablissement de l'état normal initial. Il est donc nécessaire d'associer aux aimants mixtes dans lesquels sont stockées des énergies magnétiques élevées, des dispositifs de protection élaborés.

2.1 DÉTECTION D'UNE ZONE NORMALE PAR TENSION DIFFÉRENTIELLE. - L'apparition d'une zone normale est détectée au moyen d'un montage en pont (Fig. 1). Une tension prélevée vers le milieu de la bobine est comparée à celle fournie par un diviseur résistif branché aux bornes de la bobine. Ce diviseur est réglé de telle manière que le pont soit équilibré. Dans ces conditions, le système de protection ne reçoit aucun signal lorsqu'une tension est appliquée à la bobine ; par contre, l'apparition d'une zone normale déséquilibre le pont, lequel délivre un signal proportionnel au produit de la résistance de la partie normale par le courant circulant dans la bobine.

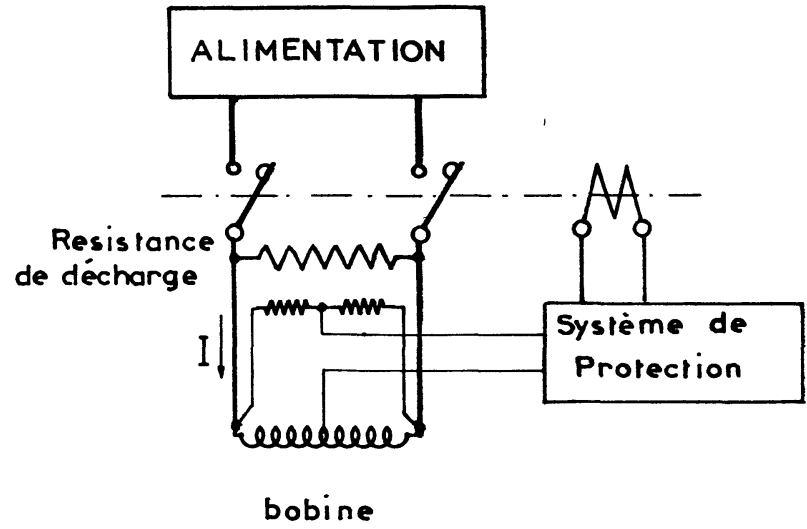

Fig. 1. - Schéma de principe de la détection d'une zone normale.

En fonction de ce signal et selon un certain critère, le système de protection commande l'ouverture du contacteur pour décharger la bobine dans une résistance (Fig. 1).

2.1.1 Critère de commande de la décharge. - Le critère le plus simple consisterait à décharger la bobine dès que le signal de déséquilibre dépasse un certain seuil. Pratiquement, ce critère est inacceptable en raison des nombreux sauts de flux se produisant durant la montée ou la descente du courant, lesquels donnent naissance à de fortes impulsions durant parfois quelques dizaines de millisecondes (Fig. 2).

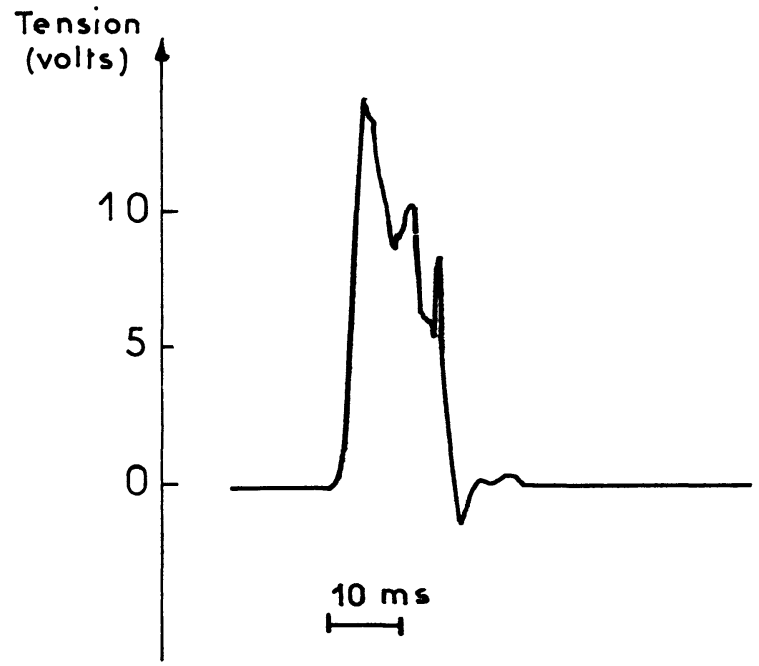

FIG. 2. - Allure de la tension induite par un saut de flux. 
Pour éviter de déclencher sur ces impulsions, la solution la plus immédiate consiste à placer un filtre du type passe-bas avant le circuit à seuil. Compte tenu de l'amplitude et de la durée des impulsions, ce système n'est pas satisfaisant car il conduit à utiliser soit des constantes de temps trop grandes, retardant exagérément l'ouverture du contacteur, soit des seuils de tension trop élevés, ne permettant pas d'assurer une protection efficace.

Finalement, le critère de décharge retenu peut s'énoncer ainsi :

L'ouverture du contacteur est commandée si la tension détectée demeure supérieure à un seuil $S$ durant un intervalle de temps supérieur à $T$.

La valeur du seuil $S$ est évidemment la plus petite possible tout en restant supérieure aux diverses tensions résiduelles (résidu d'équilibrage du pont, dérives des amplificateurs, etc...), elle est ajustable de 0,25 à $0,5 \mathrm{~V}$. Celle de $T$ est à la fois inférieure au retard maximum admissible pour l'ouverture du contacteur et supérieure à la durée des sauts de flux les plus longs ; elle est réglable de 0 à $200 \mathrm{~ms}$.

2.1.2 Fonctionnement du circuit. - Le principe de fonctionnement du circuit de détection de l'apparition d'une zone normale peut être décrit au moyen du schéma synoptique de la figure 3. Après amplification, la tension détectée est redressée puis comparée à un seuil $S$; lorsqu'il est franchi, un générateur de rampe est déclenché. Si la tension de déséquilibre persiste, la tension de la rampe atteint le niveau d'un second seuil représentant la valeur de $T$ et l'ouverture des contacteurs est alors commandée.

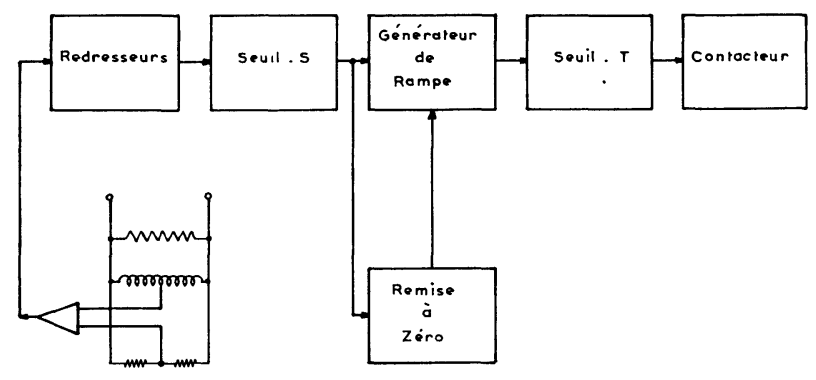

FIG. 3. - Schéma synoptique d'un détecteur de transition à tension différentielle.

Lorsque la tension détectée est induite par un saut de flux, le seuil $S$ est toujours franchi mais la rampe ne parvient pas au niveau du seuil $T$ car la durée des sauts de flux est trop courte.

Dès que la tension redressée retombe en dessous du seuil $S$, le générateur de rampe est remis à zéro afin que l'arrivée d'un autre saut de flux n'entraîne pas le dépassement du seuil $T$. En fait, cette remise à zéro est légèrement retardée pour qu'elle ne se produise pas lors d'un éventuel changement de signe de la tension détectée, à l'instant du passage à zéro. Le schéma des circuits électroniques est représenté figure 4. Les fonctions d'amplification, de redressement, de générateur de rampe et le seuil $T$ sont réalisés au moyen d'amplificateurs opérationnels. L'amplificateur différentiel d'entrée est précédé d'un circuit limiteur à diodes pour éviter la détérioration de l'amplificateur opérationnel par les fortes impulsions engendrées par les sauts de flux. Le générateur de rampe est constitué par un amplificateur monté en intégrateur; il est remis à zéro au moyen d'un transistor à effet de champ utilisé en interrupteur. La remise à zéro est retardée au moyen du réseau $\mathrm{RC}$; la diode $\mathrm{D}$ supprime le retard au déclenchement de la rampe. Pour avoir une plus grande sûreté de fonctionnement, deux circuits de ce type sont montés sur chaque bobine.

2.2 DÉTECTION PAR LA CHUTE DU COURANT. Lorsque la zone normale se propage rapidement, le courant décroît très vite ; dans ces conditions, il est possible de détecter la transition par la mesure de la chute du courant. Dans certains cas, cette chute est si brutale que ce mode de détection est plus rapide que le précédent. L'énergie libérée dans les aimants peut donc être réduite en déchargeant plus tôt les bobines.

2.2.1 Fonctionnement $d u$ circuit. - Le signal provenant du shunt de mesure du courant est amplifié puis filtré (Fig. 5). Les fréquences basses et élevées sont éliminées pour que le circuit reste insensible aux variations lentes du courant et aux diverses tensions alternatives parasites (bande passante 0,2 à $50 \mathrm{~Hz}$ ). Lors d'une chute brutale du courant, ce circuit délivre une tension dont l'amplitude initiale est proportionnelle à cette chute ; ce signal est comparé à un seuil qui, lorsqu'il est franchi, commande l'ouverture des contacteurs. Ce seuil peut être réglé de 0 à $10 \mathrm{~A}$ au moyen d'un potentiomètre. Pratiquement, il doit être réglé à quelques ampères au moins, car les sauts de flux créent de petites variations brutales du courant de l'ordre de l'ampère.

2.3 Circuits Complémentaires. - 2.3.1 Test de bon fonctionnement. - Un circuit permet de tester le bon fonctionnement du détecteur à tension différentielle avant chaque montée de champ (Fig. 4). Il est constitué par un monostable qui délivre des impulsions d'amplitude et de durée légèrement supérieures à $S$ et $T$ respectivement.

Ces impulsions sont commutées successivement sur chacune des entrées du détecteur et doivent entraîner le déclenchement.

2.3.2 Contrôle d'isolement. — La mise à la masse de deux fils de prise de tension de la bobine peut entraver le fonctionnement des détecteurs. Un circuit contrôle en permanence l'isolement des bobines; une lampe témoin s'allume dès qu'un défaut de ce type se produit (Fig. 4).

2.3.3 Tableau de lampes témoins. - Lorsqu'une disjonction se produit, un dispositif à lampes témoins indique lé détecteur qui a commandé l'ouverture des contacteurs. Les sorties des différents seuils $T$ et $\Delta I$ 


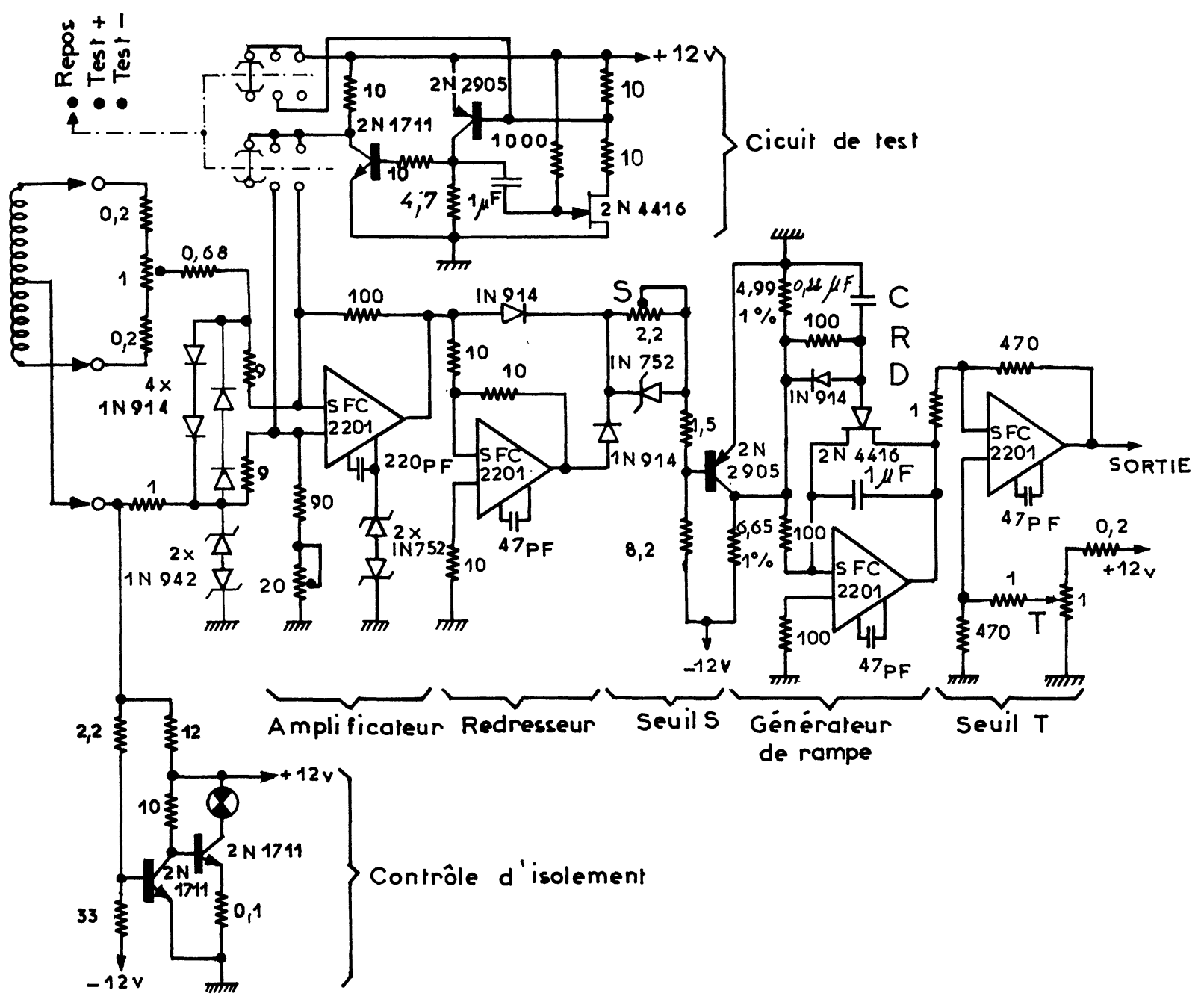

Fig. 4. - Schéma électronique d'un détecteur de transition à tension différentielle (résistances en $\mathrm{k} \Omega$ ).

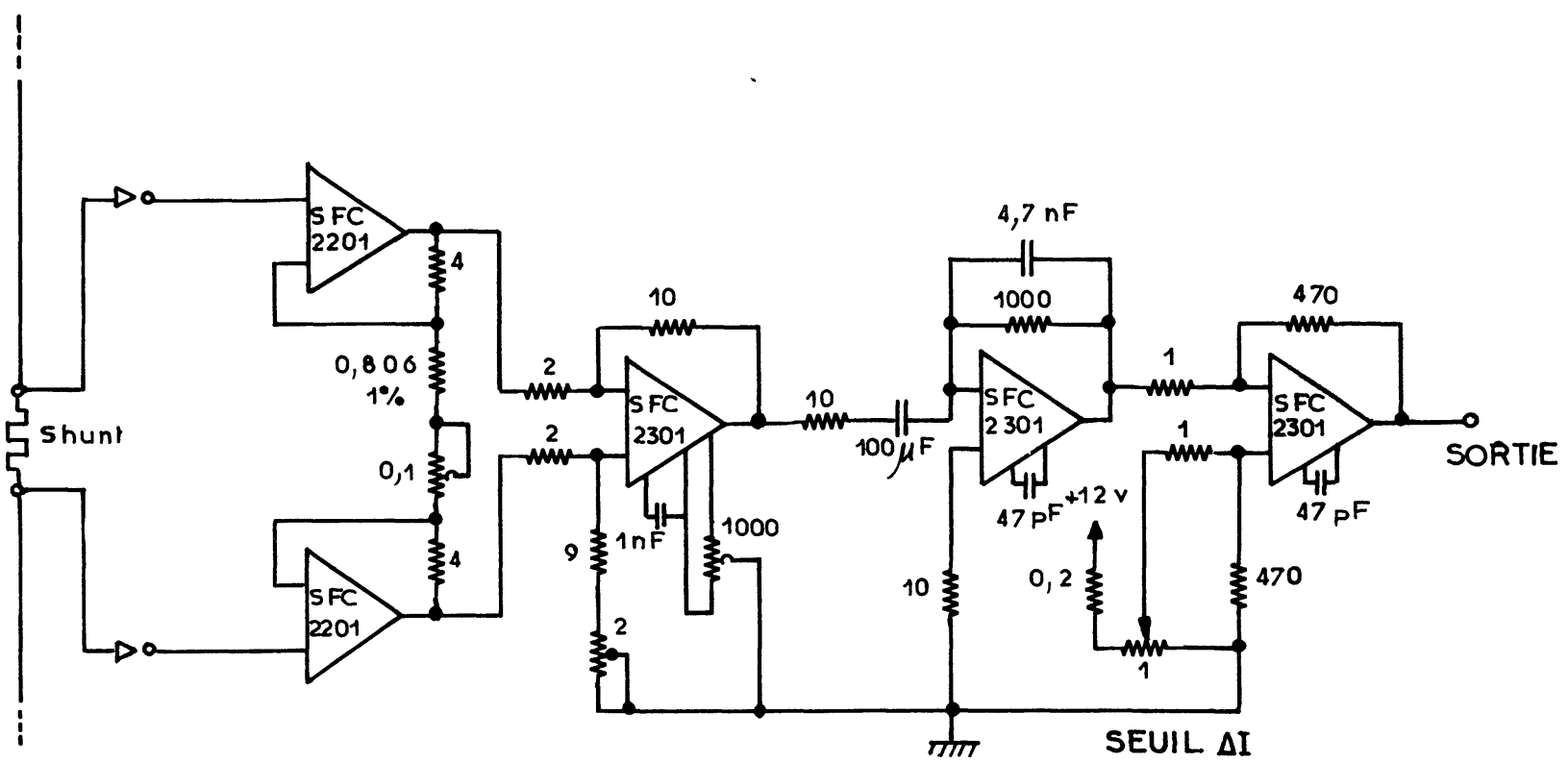

FIG. 5. - Schéma électronique d'un détecteur de transition sensible à la chute de coura nt (résistances en $\mathbf{k} \Omega$ ). 


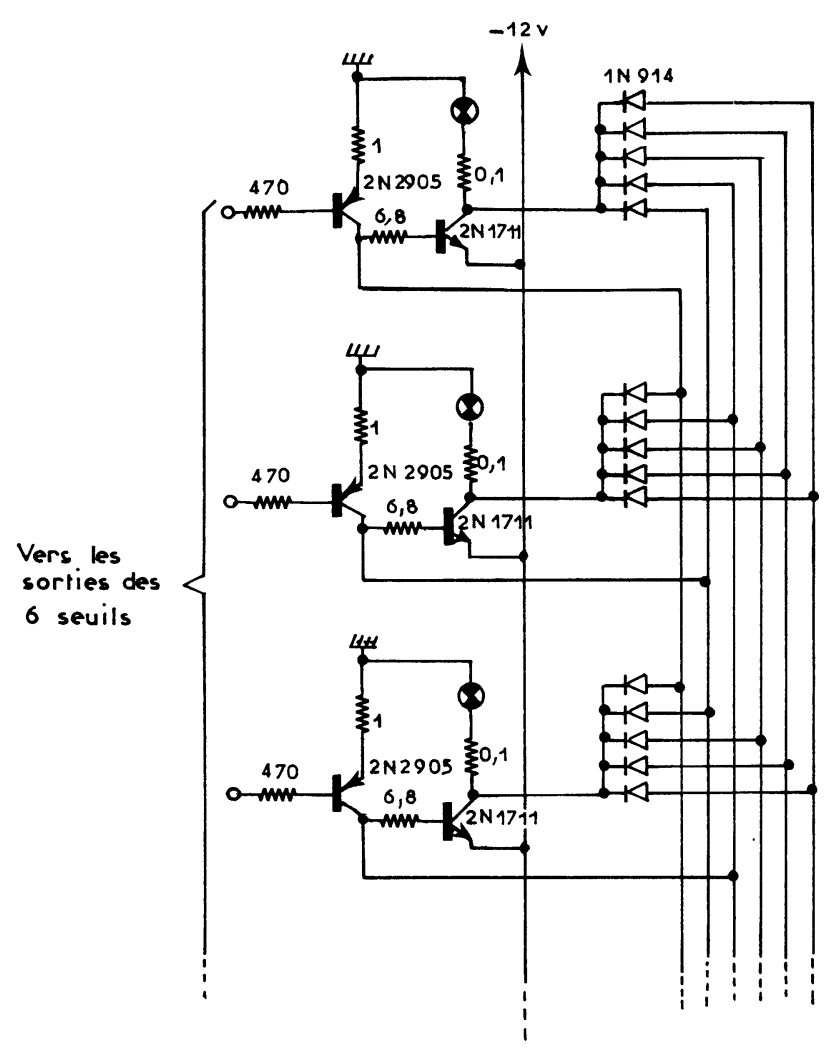

FIG. 6. - Circuit de commande des lampes témoins. sont reliées à un circuit logique (Fig. 6). Dès qu'un seuil est franchi, la lampe correspondante s'allume; un circuit de diodes interdit l'allumage de toutes les autres.

2.4 FonCtionnement D'ENSEMBle. - Le fonctionnement d'ensemble peut être décrit au moyen du diagramme représenté figure 7 . Chaque bobine possède ses propres dispositifs de protection constitués par un double circuit de détection par tension différentielle et un circuit de détection par la chute du courant. Les sorties des différents seuils $T$ et $\Delta I$ commandent l'ouverture des contacteurs par l'intermédiaire de relais. Dès qu'un seuil est franchi, les contacteurs des deux bobines sont ouverts simultanément.

3. Application à un aimant mixte 14 T. - Un aimant mixte destiné à produire un champ magnétique élevé a été construit. L'enroulement extérieur est réalisé en fil $\mathrm{Nb}$-Ti de rapport cuivre/supraconducteur égal à 5,5 fabriqué par la Société Thomson. Le courant de fonctionnement de l'aimant à $4,2 \mathrm{~K}$ est $255 \mathrm{~A}$ $(H / I=225 \mathrm{Oe} / \mathrm{A})$, la self de l'aimant est 8,08 $\mathrm{H}$. L'enroulement intérieur est réalisé en ruban $\mathrm{Nb3}-\mathrm{Sn}$ Thomson. Deux types de rubans sont employés, le rapport entre section de cuivre et section totale de matériau est de l'ordre de $\frac{1}{3}$ et $\frac{2}{3}$ pour l'un et l'autre

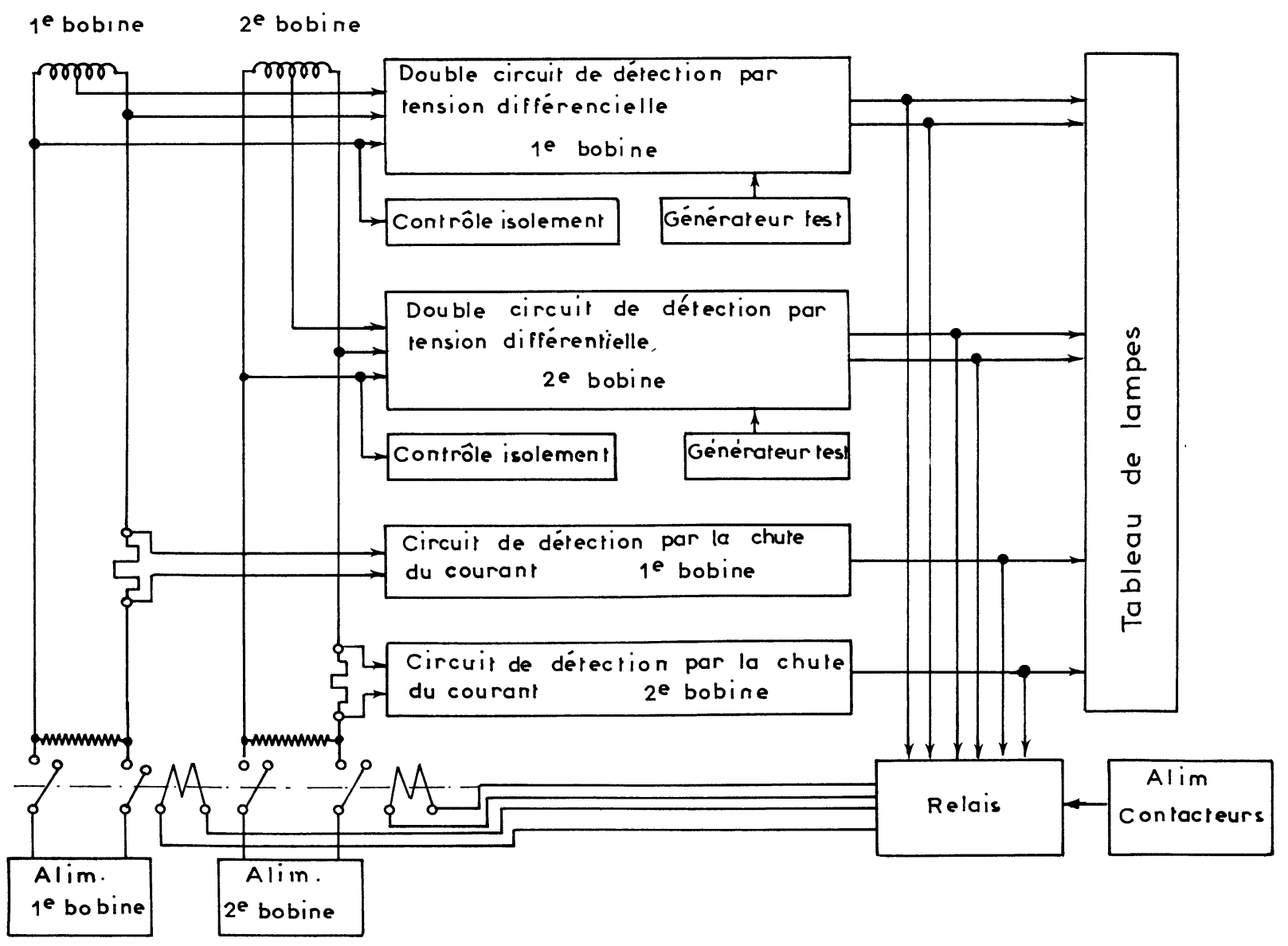

Fig. 7. - Diagramme d'ensemble. 
type. Le courant dans l'aimant est de l'ordre de $150 \mathrm{~A}$ à $4,2 \mathrm{~K}(H / I=545 \mathrm{Oe} / \mathrm{A})$. La self de l'aimant est $2,47 \mathrm{H}$. Le coefficient de couplage entre les deux bobinages est 0,45 . Les valeurs indiquées ici sont des valeurs d'inductance et de coefficient d'induction mutuelle calculés en négligeant la contribution des éléments de volume supraconducteur et sont en concordance avec les mesures faites ultérieurement. Le bilan d'énergie magnétique dans le dispositif est alors de l'ordre de $370 \mathrm{~kJ}$ à $4,2 \mathrm{~K}$, pour un champ total produit de $14 \mathrm{~T}$.

En cas de transition, les densités de courant dans les stabilisants associés aux différents matériaux sont telles que l'enroulement le plus susceptible de détérioration est celui réalisé en $\mathrm{Nb3}-\mathrm{Sn}$. Il faut donc faire décroître le courant le plus rapidement possible dans cet enroulement, pour y réduire l'échauffement. $R_{\mathrm{e} 1}$, la résistance de décharge associée à cet aimant aura donc une valeur élevée, seulement limitée par le niveau de surtension toléré aux bornes de l'aimant. $L_{1} / R_{\mathrm{e} 1}$ a été pris égal à $1 \mathrm{~s}$; le rapport des courants $I_{02} / I_{01}$ étant de l'ordre de 1,7 on peut calculer pour les deux bobinages les élévations de température $T_{\mathrm{f} 1}$ et $T_{\mathrm{f} 2}$. Les valeurs données au tableau $\mathrm{I}$ ont été définies pour diverses constantes de temps $L_{2} / R_{\mathrm{e} 2}$ du circuit $\mathrm{Nb}$-Ti. Elles sont obtenues en supposant que le rétablissement de l'état normal est simultané dans les deux bobinages, que le déclenchement des systèmes de protection est effectif à l'instant initial où s'amorce la transition; nous avons également supposé que les résistances d'enroulement restent toujours petites devant les résistances $R_{\mathrm{e} 1}$ et $R_{\mathrm{e} 2}$.

\section{TABleau I}

Les valeurs de $T_{\mathrm{f} 1}, T_{\mathrm{f} 2}$, de $t_{01}$ et $t_{02}$ ont été déterminées pour $L_{1} / R_{\mathrm{e} 1}=1 \mathrm{~s}$, avec $I_{01}=150 \mathrm{~A}$ et $I_{02}=255 \mathrm{~A}$

$\begin{array}{lcrrrr}L_{2} / R_{\mathrm{e} 2} & \mathrm{~S} & 3 & 4 & 5 & 6 \\ T_{\mathrm{f} 1} & \mathrm{~K} & 145 & 122 & 110 & 100 \\ T_{\mathrm{f} 2} & \mathrm{~K} & 58 & 68 & 80 & 92 \\ t_{01} & \mathrm{~ms} & 175 & 215 & 242 & 261 \\ t_{02} & \mathrm{~S} & 5,6 & 5,0 & 4,4 & 3,9\end{array}$

En pratique, il est indispensable de différer le déclenchement des systèmes de protection. En considérant que l'élévation de température maximale admissible ne doit pas amener certains éléments de volume de matériau au-delà de $300 \mathrm{~K}$, si $T_{\mathrm{f} 1}$ et $T_{\mathrm{f} 2}$ sont inférieures à $300 \mathrm{~K}$ elles-mêmes, on peut définir les délais de déclenchement $t_{01}$ et $t_{02}$ que l'on peut « afficher » sur l'électronique de protection décrite précédemment. Les valeurs qui figurent dans le tableau I traduisent bien la plus grande fragilité de l'enroulement $\mathrm{Nb}_{3}-\mathrm{Sn}$. Pour augmenter $t_{01}$, il faut ralentir la décroissance du cou rant dans le bobinage $\mathrm{Nb}-\mathrm{Ti}$, et on constate que l'on peut associer sans danger au circuit de cet aimant une constante de temps supérieure en durée à $6 \mathrm{~s}$, à $4,2 \mathrm{~K}$. Cependant, parce que le fonctionnement du bobinage mixte à $2 \mathrm{~K}$ avait été envisagé, et pour tenir compte de l'accroissement sensible de l'énergie magnétique stockée dans le dispositif à cette température, une constante de temps de 4,5 s propre au circuit de l'aimant $\mathrm{Nb}$-Ti a finalement été choisie.

$\mathrm{Au}$ moment des transitions que nous avons observées, nous avons constaté que la résistance de l'enroulement $\mathrm{Nb} 3-\mathrm{Sn}$ ne restait pas complètement négligeable devant $R_{\mathrm{e} 1}$, ce qui a pour effet de réduire sensiblement la température réellement atteinte dans le bobinage $\mathrm{Nb3-Sn} \mathrm{par} \mathrm{rapport} \mathrm{à} \mathrm{la} \mathrm{valeur} \mathrm{calculée.} \mathrm{Les} \mathrm{délais}$ admis ont été fixés à $100 \mathrm{~ms}$ pour le circuit Nb3-Sn, et à $400 \mathrm{~ms}$ environ dans le circuit de l'aimant $\mathrm{Nb}$-Ti.

4. Conclusion. - Nous avons examiné les problèmes posés par la protection rigoureuse d'un ensemble couplé de deux aimants supraconducteurs. Nous avons montré qu'il était possible de définir des dispositifs de protection externes aux aimants, capables d'éviter à ceux-ci des détériorations en cours de transition. L'appareil décrit ici a été mis au point au Centre de Recherches sur les Très Basses Températures à Grenoble, et les essais successifs ont confirmé la fiabilité de fonctionnement de cet appareil. L'emploi d'alliages $\mathrm{Nb}$ - $\mathrm{Ti}$ à structure multifilamentaire devrait désormais permettre la réalisation d'aimants mixtes assez maniables, mais qui resteront évidemment toujours d'emploi complexe, comparés aux aimants simples. Dans la mesure où sont d'ores et déjà commercialisés des aimants simples produisant 15 ou $16 \mathrm{~T}$, les dispositifs mixtes conservent un intérêt dans la mesure où ils permettent une économie d'investissement appréciable. Cela semble être le cas lorsque l'on veut produire des champs magnétiques supérieurs à la douzaine de tesla dans de grands volumes ; cela pourrait devenir le cas lorsque pour mettre en œuvre dans un avenir assez proche des bobinages produisant $18 \mathrm{~T}$ ou plus, les techniciens chercheront à combiner avec $\mathrm{Nb3}-\mathrm{Sn}$, un matériau comme V3-Ga, ou même d'autres composés non encore commercialisés à ce jour.

Remerciements. - Nous tenons à exprimer notre reconnaissance à $\mathbf{M}$. A. Blanchard, du laboratoire de Magnétisme (C. N. R. S. Grenoble) pour son amicale collaboration et le Laboratoire d'Electronique et de Technologie de l'Informatique (CEA Grenoble) pour son aide technique précieuse lors de la réalisation des détecteurs de transition.

\section{Bibliographie}

[1] Contrat D. G. R. S. T., 69-02-014 « Electrotechnique Nouvelle $»$.

[2] Sмiтh, P. F., Rev. Sci. Instrum. 34 (1963) 368.

[3] DESPORTES, H., International Conference on Magnet Technology, Oxford 1967, 603.
[4] Bronca, G., Krikorian, J., Néel, J., Vassal, G., BurNIER, P., MOREAU, J., Nucl. Instrum. Methods 79 (1970) 309. 